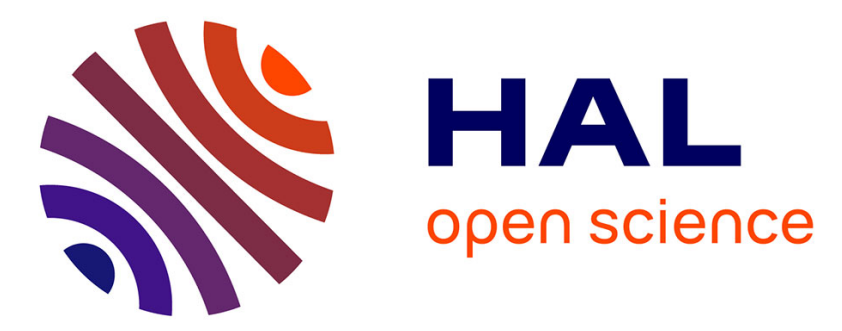

\title{
Magneto-transport properties of a random distribution of few-layer graphene patches.
}

Fabrice Iacovella, Pierre Trinsoutrot, Anatolie Mitioglu, Véronique Conédéra, Mathieu Pierre, Bertrand Raquet, Michel Goiran, Hugues Vergnes, Brigitte Caussat, Paulina Plochocka, et al.

\section{To cite this version:}

Fabrice Iacovella, Pierre Trinsoutrot, Anatolie Mitioglu, Véronique Conédéra, Mathieu Pierre, et al.. Magneto-transport properties of a random distribution of few-layer graphene patches.. Journal of Applied Physics, 2014, 116 (19), pp.1-7. 10.1063/1.4901953 . hal-01699088

\section{HAL Id: hal-01699088 https://hal.science/hal-01699088}

Submitted on 11 Jan 2022

HAL is a multi-disciplinary open access archive for the deposit and dissemination of scientific research documents, whether they are published or not. The documents may come from teaching and research institutions in France or abroad, or from public or private research centers.
L'archive ouverte pluridisciplinaire HAL, est destinée au dépôt et à la diffusion de documents scientifiques de niveau recherche, publiés ou non, émanant des établissements d'enseignement et de recherche français ou étrangers, des laboratoires publics ou privés. 


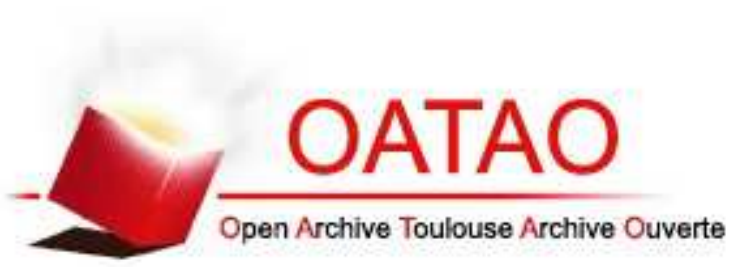

\section{Open Archive TOULOUSE Archive Ouverte (OATAO)}

OATAO is an open access repository that collects the work of Toulouse researchers and makes it freely available over the web where possible.

This is an author-deposited version published in : http://oatao.univ-toulouse.fr/ Eprints ID : 13310

To link to this article : doi: $10.1063 / 1.4901953$

URL : http://dx.doi.org/10.1063/1.4901953

To cite this version : Iacovella, Fabrice and Trinsoutrot, Pierre and Mitioglu, Anatoli and Conédéra, Véronique and Pierre, Matthieu and Raquet, Bertrand and Goiran, Michel and Vergnes, Hugues and Caussat, Brigitte and Plochoka, Paulina and Escoffier, Walter Magneto-transport properties of a random distribution of few-layer graphene patches. (2014) Journal of Applied Physics, Vol. $116\left(\mathrm{n}^{\circ}\right.$ 19). pp. 1-7. ISSN 0021-8979

Any correspondance concerning this service should be sent to the repository administrator: staff-oatao@ listes-diff.inp-toulouse.fr 


\title{
Magneto-transport properties of a random distribution of few-layer graphene patches
}

\author{
Fabrice lacovella, ${ }^{1}$ Pierre Trinsoutrot, ${ }^{2}$ Anatolie Mitioglu, ${ }^{1}$ Véronique Conédéra,,${ }^{3,4}$ \\ Mathieu Pierre, ${ }^{1}$ Bertrand Raquet, ${ }^{1}$ Michel Goiran, ${ }^{1}$ Hugues Vergnes, ${ }^{2}$ Brigitte Caussat, ${ }^{2}$ \\ Paulina Plochocka, ${ }^{1}$ and Walter Escoffier ${ }^{1, a)}$ \\ ${ }^{1}$ Laboratoire National des Champs Magnétiques Intenses, University of Toulouse, UPS, INSA, \\ CNRS-UPR 3228, 143 av. de Rangueil, F-31400 Toulouse, France \\ ${ }^{2}$ Laboratoire de Génie Chimique, ENSIACETIINP de Toulouse, UMR CNRS 5503, 4 allée Emile Monso, \\ 31432 Toulouse, France \\ ${ }^{3}$ CNRS, LAAS, 7 avenue du colonel Roche, F-31400 Toulouse, France \\ ${ }^{4}$ University of Toulouse, LAAS, F-31400 Toulouse, France
}

\begin{abstract}
In this study, we address the electronic properties of conducting films constituted of an array of randomly distributed few layer graphene patches and investigate on their most salient galvanometric features in the moderate and extreme disordered limit. We demonstrate that, in annealed devices, the ambipolar behaviour and the onset of Landau level quantization in high magnetic field constitute robust hallmarks of few-layer graphene films. In the strong disorder limit, however, the magnetotransport properties are best described by a variable-range hopping behaviour. A large negative magneto-conductance is observed at the charge neutrality point, in consistency with localized transport regime.
\end{abstract}

\section{INTRODUCTION}

The large scale fabrication of graphene is a prerequisite for its industrial integration into complex devices, either as active or as interconnecting parts of electronic circuits. ${ }^{1}$ For this purpose, the Chemical Vapor Deposition (CVD) of graphene is nowadays the most promising technological solution for producing large areas of polycrystalline graphene films. This technique, however, requires a costly state-of-the-art control of the whole fabrication process in order to achieve the highest quality specimen, ${ }^{2,3}$ where the electronic mobility can reach $5000 \mathrm{~cm}^{2} / \mathrm{Vs}$. When these drastic requirements are not fulfilled, however, one may end up with an inhomogeneous array of interconnected patches of Few Layer Graphene (FLG) with different thicknesses. Furthermore, the presence of structural defects (grain boundaries, point defects, lattice deformation, tears, edge roughness...) as well as the direct chemical environment (charge adsorbates, influence of the substrate, presence of chemical residues...) may strongly modify the performances of the pristine CVD graphene-based devices. The sensitiveness of graphene to disorder and morphology variations was realized since its infancy and many efforts have been undertaken in order to circumvent this issue. ${ }^{4-6}$ For some dedicated applications, however, lowering the production costs to the detriment of the homogeneity and electronic mobility is advantageous. It is therefore interesting to investigate on such CVD-FLG films and to provide insights on how robust are the electronic properties of such films when subject to strong roughness and disorder. In this manuscript, we focus on two CVD-FLG films deposited onto $\mathrm{Si} / \mathrm{SiO}_{2}$ substrates and integrated into FET (Field Effect Transistor) devices using simple and standard fabrication

\footnotetext{
a)walter.escoffier@lncmi.cnrs.fr
}

methods. The two devices were fabricated under the same experimental conditions, but one of them (sample S1) was annealed prior to measurement while the other one (sample S2) did not experience any post-fabrication treatment. The resulting transport properties at low temperature and high magnetic field differ substantially pointing towards the role of annealing. Indeed, while sample S1 displays well defined signatures of Landau Level (LL) quantization, sample S2 exhibits poor conduction properties and a negative magnetoconductance whose origin will be discussed.

\section{SAMPLE DESCRIPTION}

Using a 3-step process at $1000^{\circ} \mathrm{C}$, the FLG films are synthesized by catalytic CVD on copper foil $\left(2 \times 2 \mathrm{~cm}^{2}\right.$, $25 \mu \mathrm{m}$ thick, 99.999\% Alfa Aesar) from methane highly diluted into argon and hydrogen. Next, the films are transferred onto $\mathrm{Si} / \mathrm{SiO}_{2}$ substrates using a sacrificial PMMA membrane, after the copper foil has been removed using an acid solution $\left(1 \% \mathrm{HCL}+1 \% \mathrm{H}_{2} \mathrm{O}_{2}\right)$. The samples are then etched into a standard Hall bar of width $W=50 \mu \mathrm{m}$ and length $L=300 \mu \mathrm{m}$, and are electrically addressed using standard lithography methods. Sample S1 was annealed in $\mathrm{Ar} / \mathrm{H}_{2}$ at $300^{\circ} \mathrm{C}$ during two hours. Note that the devices are equipped with a back-gate of $300 \mathrm{~nm} \mathrm{SiO}_{2}$ layer that allows charge carrier density modulation through the application of a gate voltage $V_{g}$. The fabrication techniques may introduce wrinkles, impurities, tearing and other structural defects in the FLG film. Indeed, as shown in Fig. 1(c), the large ratio of Raman peaks $\mathrm{I}_{D} / \mathrm{I}_{G}$ confirms the presence of strong disorder ${ }^{7}$ at the final stage of the fabrication procedure, whereas the Dband is absent in the Raman spectra recorded before the transfer onto $\mathrm{SiO}_{2}$ (not shown) implying a very low initial defect density. ${ }^{8}$ The optical images of the devices show a 
a)

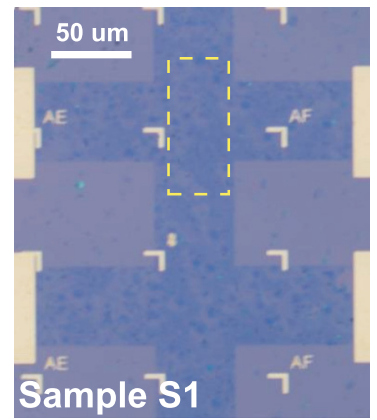

c)

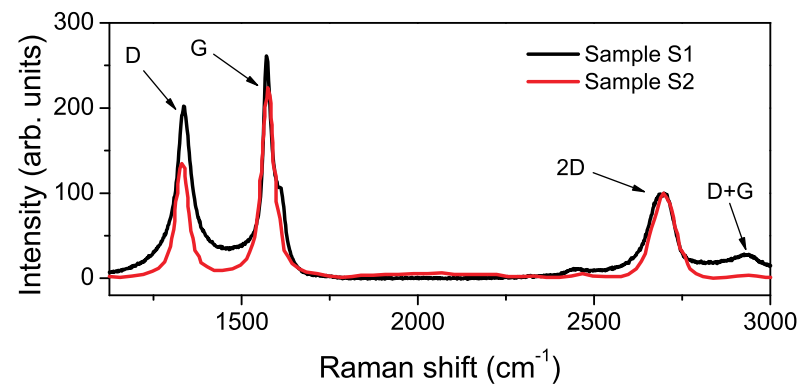

FIG. 1. (a) and (b) Optical microscope images of the devices under study. Notice the presence of macroscopic bright and dark zones on both samples, suggesting the presence of multi-layers graphene patches. The dotted-line rectangle refers to as a zone investigated in Figure 2. (c) Raman spectra for sample S1 (black curve) and sample S2 (red curve) after transfer onto $\mathrm{SiO}_{2}$ substrate. The large ratio of Raman peaks $\mathrm{I}_{G} / \mathrm{I}_{D}$ indicates a strong alteration of the graphene quality.

clear slashed structure, where patches of dark and bright spots are clearly visible (Figures 1(a) and 1(b)). Although the macroscopic details cannot be representative of the microscopic disorder, it is evident that the films are not homogeneous, but composed of an array of multi-layer graphene areas electrically connected to each other. It is worth noticing that the small ratio of Raman peaks $I_{2 D} / I_{G}<1$ adds further credits to the FLG nature of the films. Based on optical microscopy and Raman spectroscopy, the two samples are almost indistinguishable from each other despite the annealing process of sample S1. These techniques are often used to qualify graphene films from CVD processes since they constitute rapid, cheap and non-destructive characterization tools. In the present study, however, we shall show that desamples turned out to be quite different.

\section{WEAK DISORDER LIMIT}

First, we focus on sample S1. Its basic electrical characteristics are reproduced in Figure 2(a). Similar to mono-layer graphene, the resistivity of the device is maximum when the carrier density is zero on average and decreases when the sample is doped either with electrons or holes suggesting an ambipolar behavior. We note that the Charge Neutrality Point (CNP) is reached when $V_{g} \approx 10 \mathrm{~V}$, accounting for the presence of charged impurities in the close vicinity of the FLG film. As expected, the resistivity peak becomes sharper at low temperature and spans from $1.5 \mathrm{k} \Omega$ to $8 \mathrm{k} \Omega$ in the investigated range of back-gate voltage. The carrier density $n$ is extracted from low-field Hall effect measurements and displayed in Figure 2(b) for selected values of $V_{g}$. Assuming a plane spite strong similarities, the electronic properties of the two a)

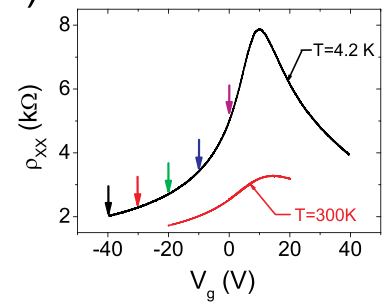

c)

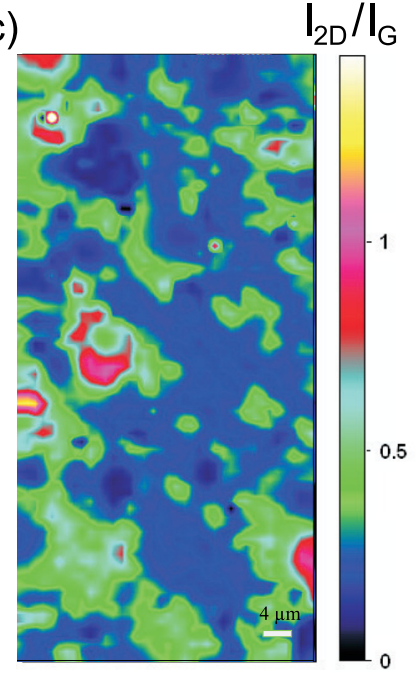

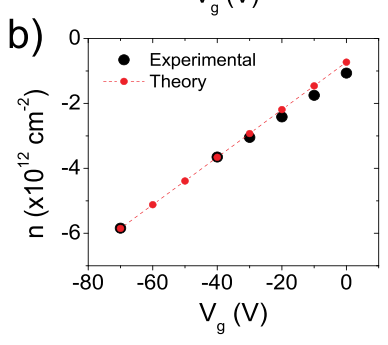

FIG. 2. (a) The ambipolar behavior of device $\mathrm{S} 1$ is indicated by the $R_{X X}\left(V_{g}\right)$ characteristic, where the culminating point represents the $\mathrm{CNP}$ at $\mathrm{T}=300 \mathrm{~K}$ and $\mathrm{T}=4.2 \mathrm{~K}$. The arrows show selected back-gate voltages at which magneto-transport measurements have been performed. (b) Evolution of the carrier density as a function of back-gate voltage: the linear behavior is favorably compared to standard plane capacitor model. (c) Raman spectroscopy map of a part of the sample S1 (see dotted-line rectangle in Figure 1(a), evidencing an inhomogeneous film made of few-layer graphene patches.

capacitor model, the linear relation $n\left(V_{g}\right)=\left[\epsilon_{0} \epsilon_{r}\left(V_{g}-\right.\right.$ $\left.\left.V_{C N P}\right)\right] /(e \times d)$ is favorably compared to the experimental values for the gate dielectric thickness $d=295 \mathrm{~nm}$ ( $e$ is the electron charge, $\epsilon_{0}$ and $\epsilon_{r}=3.9$ are, respectively, the vacuum and relative dielectric permittivity of $\mathrm{SiO}_{2}$ ). The slight deviation of the experimental data at $V_{g} \sim V_{C N P}$ is accounted by the presence of mixed charge polarity in the close vicinity of the CNP. Overall, this result indicates that the gate efficiency $\left(7.2 \times 10^{10} \mathrm{~cm}^{-2} / \mathrm{V}\right)$ is unperturbed by the presence of the multi-layer graphene patches. Indeed, screening effects usually observed in graphite and thick multi-layer graphene flakes $^{9,10}$ are absent pointing towards a film of interconnected FLG zones where the average number of graphene layers in each patch does not exceed $\sim 5 .{ }^{11-13}$ The inhomogeneous distribution of few-layer areas is further evidenced in Figure 2(c) where the ratio between the intensity of the D and G Raman peaks is spatially resolved over a limited zone of the sample (shown in Figure 1(a)). The average size of the FLG patches roughly reads $5 \mu \mathrm{m}$, in agreement with optical microscope observations.

The high field magneto-transport results are shown in Figure 3 for sample S1. A fast data acquisition system is used to record both the longitudinal and Hall resistance during a pulse of magnetic field with maximum value $B=55 \mathrm{~T}$ and of total duration $\tau \sim 300 \mathrm{~ms} .{ }^{14}$ In order to avoid longitudinal and Hall resistance mixing, each curve depicted in Figure 3 corresponds, respectively, to the sum and difference of the data recorded from a positive and negative magnetic field while the back-gate voltage is kept constant at some selected values as depicted in Figure 2(a). The mobility of the FLG film is estimated to $\mu=1100 \mathrm{~cm}^{2} / \mathrm{Vs}$ at $\mathrm{T}=4.2 \mathrm{~K}$ and at carrier density $n=3.7 \times 10^{12} \mathrm{~cm}^{-2}\left(V_{g}=-40 \mathrm{~V}\right)$. At high magnetic field, both the longitudinal (Figure 3(a)) 

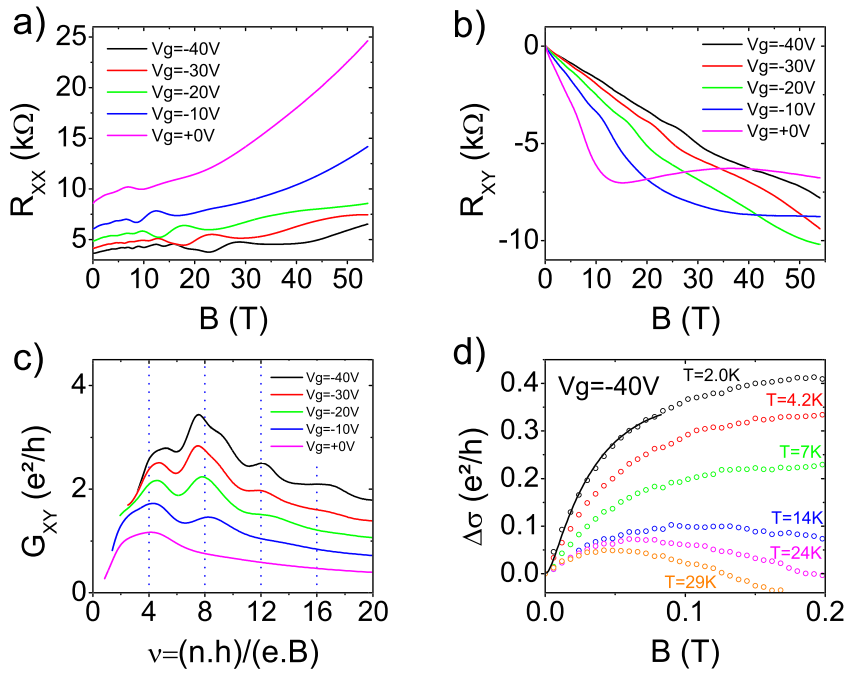

FIG. 3. (a) and (b) Four-probe longitudinal magneto-resistance $\left(R_{x x}\right)$ and Hall resistance $\left(R_{x y}\right)$, respectively, of sample $\mathrm{S} 1$ at $\mathrm{T}=4.2 \mathrm{~K}$ and for selected values of $V_{g}$. Magneto-resistance oscillations are clearly visible suggesting the onset of the quantum Hall regime. (c) Hall conductance $\left(G_{x y}\right)$ is obtained through resistance tensor inversion. (d) Weak-localization effect at various temperature for B $<0.2 \mathrm{~T}$ and $V_{g}=40 \mathrm{~V}$. The theoretical fit is displayed for $\mathrm{T}=2 \mathrm{~K}$.

and Hall resistance (Figure 3(b)) shows large oscillations, displaying an expected phase shift as the back-gate voltage is varied. The frequency of magneto-oscillations depends on the carrier density and it is often more convenient to plot the data as a function of the LL filling factor $\nu=n . h / e . B$ as shown in Figure 3(c) where the Hall conductance $G_{x y}(B)$ is calculated through resistance tensor inversion. Clearly, the observed features cannot be compared to those of mono-, biand tri-layer graphene ${ }^{15}$ of similar mobility, which would display clear conductance quantization at low filling factor. In this sample, the broad dispersion of FLG zones prevents the emergence of the Quantum Hall regime. The film should be regarded as a polycrystal, where each FLG "grain" develops its own LL energy spectrum under high magnetic field, so that the global response of the system is an incoherent manifestation of the electronics properties of each of them. Interestingly, the oscillations appear pseudo-periodic with a main period $\nu=4$. This special feature originates from the four-fold degeneracy of LLs (except for the zeroth LL) shared by at least mono-layer, bi-layer, and tri-layer graphenes $^{16,17}$ as well as weakly coupled FLG systems. It constitutes a robust feature which is not averaged out by the thickness dispersion of the FLG patches. These magnetotransport results should be compared to those of Ref. 18, where the emergence of the quantum Hall effect is observed in a graphene film decorated with multi-layer graphene areas. The authors evidenced the presence of a robust and quantized Hall resistance plateau at filling factor $\nu=2$ indicating that, provided a continuous mono-layer graphene film is connecting the device's electrodes, the inclusion of multi-layer graphene areas on top of it does not significantly modify the graphene's LL spectrum. The situation is certainly different in sample S1 where the underlying continuous graphene mono-layer is absent. For completeness, we notice that the system displays weak localization effect at low magnetic field as shown in Figure 3(d). Following the lines of Ref. 19, we extract a phase coherence length of the order of $\ell_{\varphi} \approx 200 \mathrm{~nm}$ at $\mathrm{T}=2 \mathrm{~K}$, i.e., roughly one order of magnitude lower than the estimated diameter of the FLG patches.

Another remarkable feature can be noticed at low carrier density, where the longitudinal resistance strongly increases with magnetic field. When the Fermi level is close to the $\mathrm{CNP}$, charge carriers are populating the zeroth LL whose energy remains independent of the strength of the magnetic field (spontaneous symmetry breaking effects are not taken into account since disorder prevents the observation of any spin and valley degeneracy lifting). In other words, the Fermi energy lies in an energy window with ever increasing density of states as the magnetic field increases, promoting electronic diffusion and resulting in a large positive magneto-resistance. On the other hand, the Hall resistance is not linear with B anymore at high field because of the inevitable presence of electron-hole puddles. ${ }^{20}$ The system simultaneously holds both types of charge carriers (electrons and holes) with opposite contribution to the Hall resistance. Thus, at high enough magnetic field, once the Fermi energy is pinned into the emerging zeroth LL, the saturating Hall resistance constitutes a direct indication of the ratio between electron and hole carrier densities in the system at a given back gate voltage. ${ }^{21}$ In the sample under study, the presence of many disordered FLG patches with different LL spectra and doping naturally tends to reduce the amplitude of this effect and to enlarge the energy window over which it is observed. Nevertheless, its complete vanishing is very unlikely since it relies on the emergence of a zeroth LL which, in a first approximation, is a robust property shared by all disordered FLG systems.

\section{STRONG DISORDER LIMIT}

Let us now focus on sample $\mathrm{S} 2$, which was prepared using the same recipe as sample S1 except for postfabrication annealing. Despite their similar optical microscopy and Raman spectroscopy features, the transport properties of sample S2 are much different than those reported above. First of all, the resistivity is almost two orders of magnitude larger in device $\mathrm{S} 2(100 \mathrm{k} \Omega)$ than in device $\mathrm{S} 1$ $(3 \mathrm{k} \Omega)$ at room temperature. In order to comply with high resistance value, especially at low temperature, the two-probe conductance of device S2 is measured by monitoring the current flowing from source to drain electrodes as a function of bias voltage $V_{\text {bias }}$. The I-V characteristics develop a nonlinear behaviour when $\mathrm{T}<85 \mathrm{~K}$. This effect is best evidenced in a small device (i.e., in sample $\mathrm{S} 2 *$ of length $9 \mu \mathrm{m}$ and width $5 \mu \mathrm{m}$ made from the same FLG film as sample S2) as shown in Figure 4(a). Alternatively, this trend is also visible when considering the differential conductance curves $(d I / d V)$ which were recorded simultaneously with the I-V characteristics by adding a small modulation voltage and using lock-in detection. They provide an alternative representation of the quenched conductance at low bias voltage and low temperature, as illustrated in Figure 4(b). Contrarily to sample S1 and due to severe contamination during the device fabrication 

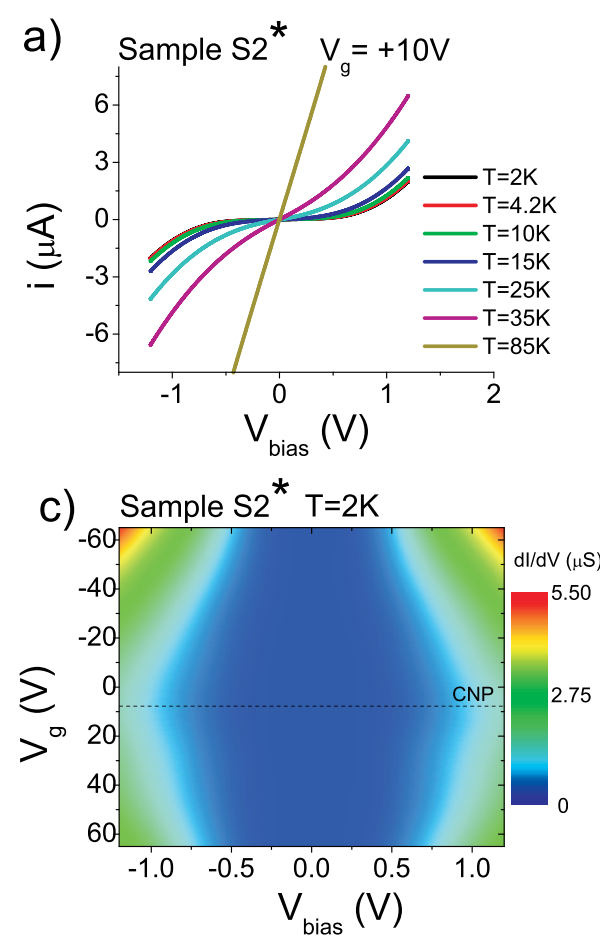

e) Sample $\mathrm{S} 2 \mathrm{~T}=25 \mathrm{~K}$

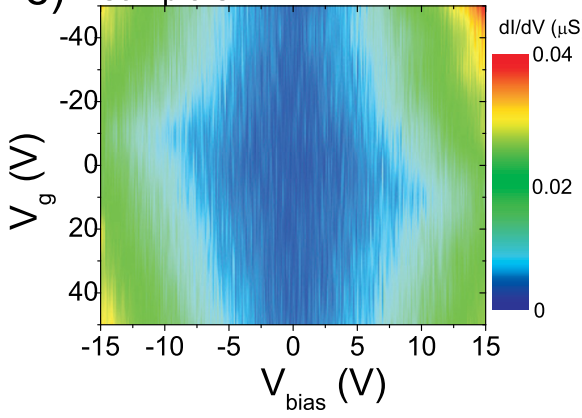

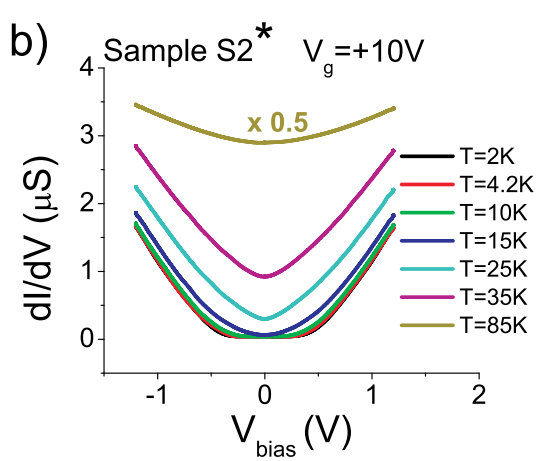
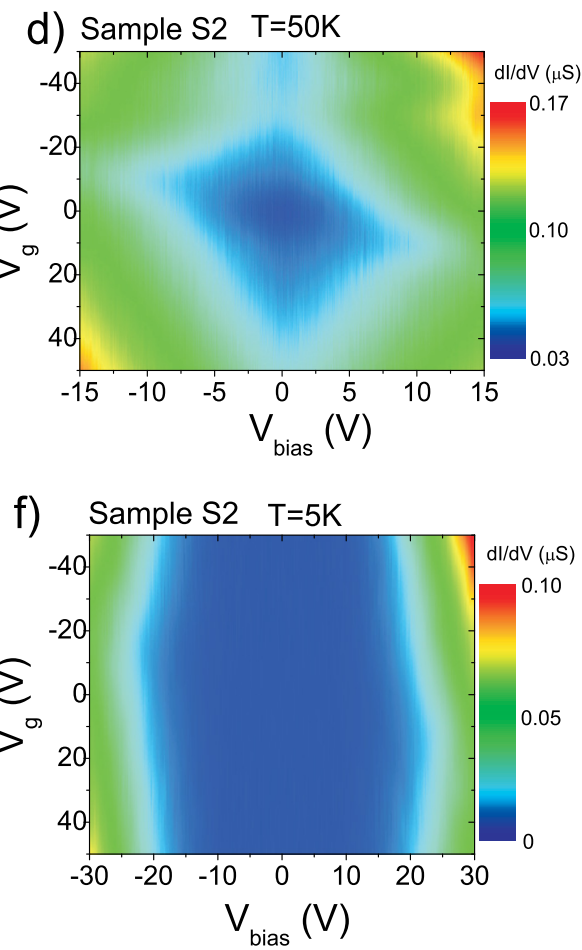

FIG. 4. (a) I-V characteristics for sample $\mathrm{S} 2 *$ from $\mathrm{T}=2 \mathrm{~K}$ to $\mathrm{T}=85 \mathrm{~K}$ : strong non-linear behavior develops at low temperature. (b) Differential conductance for sample S2*. At low bias voltage, the Coulomb blockade behavior dominates when the temperature is lower than $\sim 10 \mathrm{~K}$. (c) Color map representation of the differential conductance of sample $\mathrm{S} 2 *$, as a function of $V_{\text {bias }}$ and $V_{g}$. The graph is symmetric with respect to $V_{g}=10 \mathrm{~V}$ which indicates the CNP. (d) to (f) Color map representation of the differential conductance of sample $\mathrm{S} 2$ for $\mathrm{T}=50 \mathrm{~K}$, $25 \mathrm{~K}$, and $4.2 \mathrm{~K}$. Notice the different $V_{\text {bias }}$ scale as compared to sample $\mathrm{S} 2 *$. process, we hypothesize that this strongly disordered sample consists of many FLG islands weakly coupled to each other so that it actually behaves as a granular system with large potential barriers between the FLG grains. In the Coulomb blockade regime at low temperature, the conduction takes place through thermally activated hops of the charge carriers between neighboring FLG patches. At high bias voltage, the electrons acquire enough energy and percolate through the FLG array via multiple branching paths. The bias voltage range over which the current suppression is observed varies as a function of the system's size as well as the back-gate voltage, as shown in Figures 4(c) to 4(f), where the differential conductance as a function of $V_{\text {bias }}$ and $V_{g}$ is displayed using a color map representation. The quenched conductance effect in the small device is larger at $V_{g} \approx 10 \mathrm{~V}$ and decreases either when a positive or negative back gate voltage is applied (see Figure 4(c)). Indeed, as the system is driven close to the CNP, the FLG islands are more isolated so that the hopping processes are rarer as compared to the situation where the system holds a higher carrier density. The same trend is observed for sample S2 but the "diamondshape" picture is distorted reflecting non-symmetric I-V curves with respect to $V_{\text {bias }}=0 \mathrm{~V}$. This effect is explained through a variation in the actual gating potential across the sample, when $V_{\text {bias }}$ is of the same order of magnitude as $V_{g}$. Interestingly, even if device S2 is in a strongly localized transport regime, its sensitiveness to electrostatic gating remains and the CNP is still experimentally accessible. The color map representation of the device's characteristic allows an unambiguous determination of the CNP which would have been otherwise difficult to extract by analyzing the conductance against gate voltage alone.

The electronic transport in the non-linear regime at very low temperature is now discussed. For this purpose, the model of Middleton and Wingreen ${ }^{22}$ is appropriate and considers an array of capacitively coupled conductors. The I-V characteristics should follow the trend $i(V)=\beta \times\left(V-V_{t}\right)^{\alpha}$, where $\beta$ is a constant pre-factor, $V_{t}$ is the threshold bias voltage above which the current is no longer suppressed, and $\alpha$ is a scaling exponent which depends on the system's characteristics. In Figure 5, we plot the I-V curves recorded at different $V_{g}$ in a log-log scale with a constant threshold voltage $V_{t}=8.5 \mathrm{~V}$ extracted from the curve at $V_{g}=0 \mathrm{~V}$. We obtain a set of linear curves (for sufficiently high bias voltage) with similar slopes $\alpha=2.9$, but different offsets depending on $V_{g}$. Such offsets correspond to variations of $V_{t}$ as compared to its reference 
Sample 2, $\mathrm{T}=4.2 \mathrm{~K}$

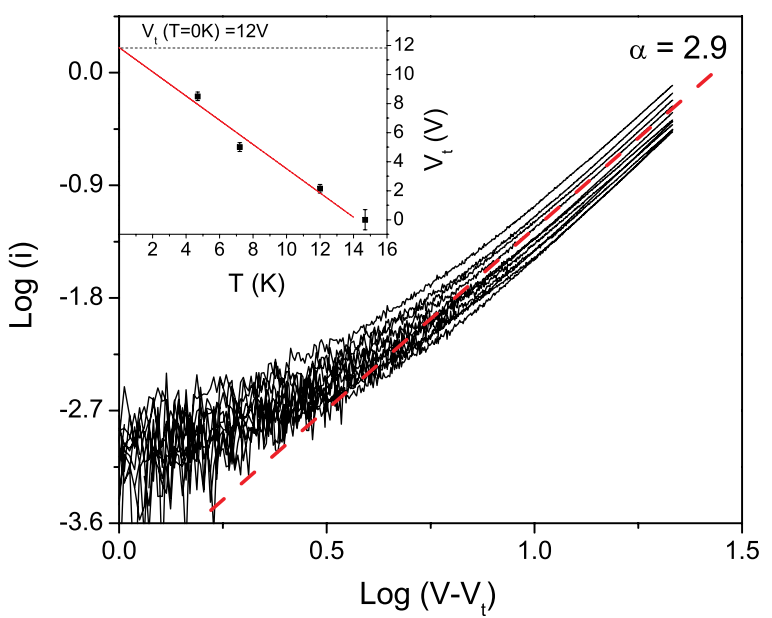

FIG. 5. I-V characteristics of sample 2 plotted in a log-log scale for backgate voltage ranging from $V_{g}=-50 \mathrm{~V}$ to $V_{g}=+50 \mathrm{~V}$. The common slope of the set of curves is indicated by the dash line. Inset: the extrapolation of the threshold voltage $V_{t}$ down to $T=0 \mathrm{~K}$ provides the global threshold voltage $V_{t}=12 \mathrm{~V}$.

value at $V_{g}=0 \mathrm{~V}$. The extracted exponent $\alpha$ is consistent with previous reports ${ }^{23}$ (and references within), which considers a random array of graphene dots. At zero gate voltage, $V_{t}$ is estimated to $8.5,5$, and $2.1 \mathrm{~V}$ at $4.2,7.5$, and $12 \mathrm{~K}$, respectively. As $V_{t}$ is expected to increase linearly with decreasing temperature, the extrapolation of the curve $V_{t}(\mathrm{~T})$ down to $T=0 \mathrm{~K}$ provides the global threshold voltage $V_{t}(T=0 \mathrm{~K}) \approx 12 \mathrm{~V}$. This parameter is directly linked to the total number (N) of FLG islands in the sample through the relation $V_{t}(T=0 \mathrm{~K}) \approx \kappa \times N \times \frac{e}{C_{0}}$, where $C_{0}=8 r \epsilon_{0} \epsilon_{r}$ represents the FLG self-capacitance (assuming that FLG islands are modeled by $2 \mathrm{D}$ disks of radius $r$ ) and $\kappa$ is a parameter that depends only on the dimensionality and the capacitive coupling $C$ between neighboring FLG islands. For a weakly coupled 2D square lattice with $C / C_{0} \ll 1$, numerical simulation provides $\kappa=0.338 .^{22}$ Alternatively, the number of quantum dots can be approached by the relation $N \times \pi \times r^{2}=L \times W$, assuming the whole area of the device is actually covered with FLG disks. Solving both equations simultaneously, we compute $r \sim 500 \mathrm{~nm}$. Even if the order of magnitude seems realistic, we emphasize that the deduction of $r$ must be handled very carefully as it relies on several initial assumptions and rough approximations. The system can thus be viewed as a granular metal with mean grain size $\sim 500 \mathrm{~nm}$ weakly coupled to each other and demonstrating a Coulomb-induced transport gap at low temperature.

Now, we would like to comment the magnetoconductance (MC) shown in Figure 6(a) for sample S2. Overall, a strong negative MC of the order of $60 \%$ is observed at $\mathrm{B}=55 \mathrm{~T}$. On the other hand, the evolution is not monotonous but first displays a positive MC reaching a maximum for $\mathrm{B} \sim 6 \mathrm{~T}$. The theory of weak localization may provide a natural explanation for such positive MC, however, this hypothesis is rapidly ruled out since (i) it usually applies in moderate disordered (diffusive) systems, (ii) it takes place within a low magnetic field range $(<1 \mathrm{~T})$, and (iii) it vanishes with increasing temperature. An alternative scenario can be a)

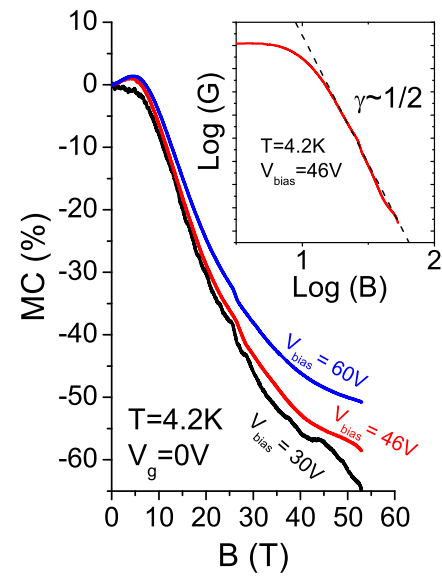

b)

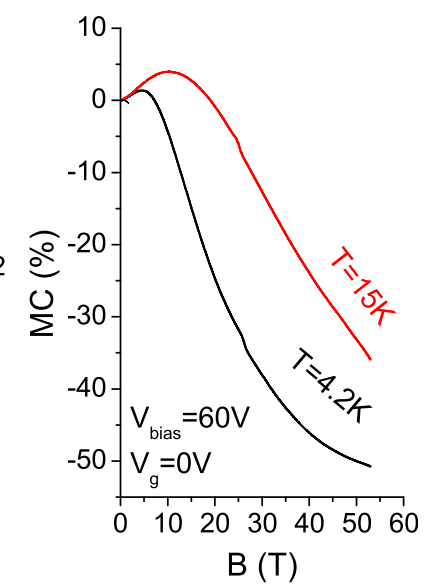

FIG. 6. (a) Magneto-conductance of sample S2 at $T=4.2 \mathrm{~K}$ and $V_{g}=0 \mathrm{~V}$ for various bias voltages. The magneto-conductance is positive up to $\sim 6 \mathrm{~T}$ before turning negative. Inset: $\log -\log$ plot of the high field magnetoconductance of $\mathrm{S} 2$, the slope provides $\gamma \approx 0.57$ for $V_{\text {bias }}=46 \mathrm{~V}$ and $T=4.2 \mathrm{~K}$. (b) Temperature dependence of the magneto-conductance of sample $\mathrm{S} 2$, showing non-monotonous behavior.

considered in order to explain the initial positive magnetoconductance: it relies on the Variable Range Hopping (VRH) model in which conduction occurs through charge hoppings between localized sites, in the strong disorder limit. Even if this model has not been initially developed for granular systems, many authors have considered it for this purpose, after they noticed an excellent agreement between the predictions of the model and the transport properties of weakly coupled nanoparticle lattices. ${ }^{24-26}$ The localization length $\xi$ and hopping length $r_{\text {hop }}$ are essential parameters of the VRH model, which are associated to the mean nanoparticle's size and their spatial separation, respectively. In sample S2, such details are not directly accessible (or deduced with a large incertainty) so that we will discuss the VRH model within a qualitative framework only. In the strongly localized regime, the hopping probability is computed through the coherent sum over all possible paths connecting two sites and thus depends on the magnetic field. ${ }^{27,28}$ A weak positive MC saturating near $B^{*}=\Phi_{0} \times r_{\text {hop }}^{-3 / 2} \times \xi^{-1 / 2}$ is expected. While the localization length is temperature-independent, the hopping length is expected to decrease as the temperature increases. Consequently, $B^{*}$ is shifted towards higher value for increasing temperature, in qualitative agreement with our experimental observations (see Figure 6(b)). Furthermore, the initial positive MC amplitude is larger for increasing $V_{\text {bias }}$, reinforcing the hypothesis that hopping processes are indeed involved in the galvanometric transport properties. This observation is also consistent with Ref. 29 who reported a magnetic-field driven delocalization effect in disordered graphene only in the presence of high electric and magnetic fields. The negative magneto-conductance for $B>B^{*}$ can be accounted for by a direct consequence of shrinking of the wavefunctions of the localized states due to magnetic confinement. In the VRH-type conduction regime, the magnetoconductance is expected to behave according to the relation $\log [\sigma(B)] \propto B^{\gamma}$, where $\gamma=1 / 2$ for Mott-VRH and $\gamma=1 / 3$ for ES-VRH. When plotted in a log-log graph, a linear 
behaviour is recovered at high field with $0.46<\gamma<0.62$ for all curves, which confirms the functional form of the magneto-conductance and establishes the Mott-VRH scenario for high magnetic field ${ }^{30}$ (for clarity, only a representative curve for $V_{\text {bias }}=+46 \mathrm{~V}$ is shown in the insert of Figure 6(a). It is worth mentioning that, at zero magnetic field and in the temperature range $90 \mathrm{~K}<\mathrm{T}<290 \mathrm{~K}$, the resistance of device $\mathrm{S} 2$ strongly increases when the temperature decreases (not shown) and scales as $R \propto \exp \left[\left(T_{0} / T\right)^{p}\right]$ with $\mathrm{p}=1 / 3$ (Mott VRH) or $\mathrm{p}=1 / 2$ (ES-VRH). This behavior provides additional credits to the VRH-like transport regime, together with the large MC amplitude at low temperature. Although the VRH model allows a coherent qualitative interpretation of the experimental results in agreement with the transport regime in sample $\mathrm{S} 2$, other phenomena inherent to graphene may superimpose or compete with those described above. In particular, each FLG patch can host LL quantization under intense magnetic field provided the disorder-induced broadening of the LLs remains lower than the energy gap between them. Although this last assumption cannot be directly checked for sample S2, some authors ${ }^{31}$ reported on the onset of the $n=0 \mathrm{LL}$ in hydrogenated graphene with mobility less than $10 \mathrm{~cm}^{2} / \mathrm{Vs}$, arising from the large energy gap between $n=0$ and $n= \pm 1$ LLs $\left(\Delta E=v_{F} \times \sqrt{2 \hbar e B}=270 \mathrm{meV}\right.$ at $55 \mathrm{~T})$. The onset of LLs, peculiar to each FLG patches, is likely to favor negative MC under high magnetic field. This assertion can be further reinforced by performing high field MC studies as the system is progressively driven far from CNP which, regrettably, could not be performed because of sample failure during the course of the experiment.

\section{SUMMARY AND REMARKS}

To summarize, two CVD-FLG films have been investigated. One of them is composed of FLG islands well interconnected to each other, while they appear separated by strong potential barriers in the second one. Since the FLG films were grown using the same experimental conditions, we suppose that the transfer from the copper catalyst to the $\mathrm{SiO}_{2}$ substrate before device processing as well as postfabrication annealing are likely to account for such a discrepancy. At low temperature, the devices display much different transport properties but both remain sensitive to electrostatic doping. The galvanometric properties show positive magneto-resistance at high magnetic field and close to the CNP. This effect is likely to originate from the onset of the $n=0 \mathrm{LL}$ which remains robust even in the presence of very strong disorder. To some extent, this study can be compared with recent reports involving functionalized graphene (graphane, graphene oxide, chlorinated graphene, fluorinated graphene are few examples), which aims at tailoring the electronic properties of graphene at wish. ${ }^{23,32-35}$ Even if the perspective of a gap opening in the density of states of such systems holds great promises for electronic applications, chemical functionalization ${ }^{36}$ inevitably introduces defects in the initial material and it is instructive to compare the resulting conduction properties with low-cost CVD graphene film containing random and uncontrolled disorder instead.

\section{ACKNOWLEDGMENTS}

CVD-FLG films were fabricated at LGC, while device preparation was carried out at LAAS. Low temperature and high magnetic field measurements were performed at LNCMI. This work was supported by EuroMagNet (Contract No. 228043) under proposal TSC33-112; by the FP7-NMP Grenada project for the CVD part and by the French RENATECH network for device processing.

${ }^{1}$ P. Avouris and C. Dimitrakopoulos, "Graphene: synthesis and applications," Mater. Today 15, 86-97 (2012).

${ }^{2}$ X. Li, W. Cai, J. An, S. Kim, J. Nah, D. Yang, R. Piner, A. Velamakanni, I. Jung, E. Tutuc, S. K. Banerjee, L. Colombo, and R. S. Ruoff, "Largearea synthesis of high-quality and uniform graphene films on copper foils," Science 324, 1312 (2009).

${ }^{3}$ M.-Y. Li, C.-C. Tang, D. C. Ling, L. J. Li, C. C. Chi, and J.-C. Chen, "Charged impurity-induced scatterings in chemical vapor deposited graphene," J. Appl. Phys. 114, 233703 (2013).

${ }^{4}$ C. R. Dean, A. F. Young, I. Meric, C. Lee, L. Wang, S. Sorgenfrei, K. Watanabe, T. Taniguchi, P. Kim, K. L. Shepard, and J. Hone, "Boron nitride substrates for high-quality graphene electronics," Nat. Nanotechnol. 5, 722-726 (2010).

${ }^{5}$ K. Bolotin, K. Sikes, J. Hone, H. Stormer, and P. Kim, "Temperaturedependant transport in suspended graphene," Phys. Rev. Lett. 101, 096802 (2008).

${ }^{6}$ X. Du, I. Skacho, A. Barker, and E. Andrei, "Approaching ballistic transport in suspended graphene," Nat. Nanotechnol. 3, 491 (2008).

${ }^{7}$ A. Ferrari, J. Meyer, V. Scardaci, C. Casiraghi, M. Lazzeri, F. Mauri, S. Piscanec, D. Jiang, K. Novoselov, S. Roth, and A. Geim, "Raman spectrum of graphene and graphene layers," Phys. Rev. Lett. 97, 187401 (2006).

${ }^{8}$ P. Trinsoutrot, C. Rabot, H. Vergnes, A. Delamoreanu, A. Zenasni, and B. Caussat, "High quality graphene synthesized by atmospheric pressure $\{\mathrm{CVD}\}$ on copper foil," in 19th European Conference on Chemical Vapor Deposition (EuroCVD19), Varna, Bulgaria, 1st-6th September 2013 [Surf. Coat. Technol. 230, 87-92 (2013)].

${ }^{9}$ A. Reina, X. Jia, J. Ho, D. Nezich, H. Son, V. Bulovic, M. S. Dresselhaus, and J. Kong, "Large area, few-layer graphene films on arbitrary substrates by chemical vapor deposition," Nano Lett. 9, 30-35 (2009).

${ }^{10}$ Y. Zhang, J. Small, W. Pontius, and P. Kim, "Fabrication and electric field-dependent transport measurements of mesoscopic graphite devices," Appl. Phys. Lett. 86, 073104 (2005).

${ }^{11} \mathrm{P}$. B. Visscher and L. M. Falicov, "Dielectric screening in a layered electron gas,” Phys. Rev. B 3, 2541-2547 (1971).

${ }^{12}$ M. Koshino, "Interlayer screening effect in graphene multilayers with $a b a$ and $a b c$ stacking," Phys. Rev. B 81, 125304 (2010).

${ }^{13}$ M. A. Kuroda, J. Tersoff, and G. J. Martyna, "Nonlinear screening in multilayer graphene systems," Phys. Rev. Lett. 106, 116804 (2011).

${ }^{14} \mathrm{~F}$. Debray and P. Frings, "State of the art and developments of high field magnets at the laboratoire national des champs magnétiques intenses," $\mathrm{C}$. R. Phys. 14, 2 (2013).

${ }^{15}$ A. Kumar, W. Escoffier, J. M. Poumirol, C. Faugeras, D. P. Arovas, M. M. Fogler, F. Guinea, S. Roche, M. Goiran, and B. Raquet, "Integer quantum hall effect in trilayer graphene," Phys. Rev. Lett. 107, 126806 (2011).

${ }^{16}$ M. Ezawa, "Intrinsic zeeman effect in graphene," J. Phys. Soc. Jpn. 76, 094701 (2007)

${ }^{17}$ S. Yuan, R. Roldán, and M. I. Katsnelson, "Landau level spectrum of aba and abc stacked trilayer graphene," Phys. Rev. B 84, 125455 (2011).

${ }^{18}$ Y. Nam, J. Sun, N. Lindvall, S. Jae Yang, D. Kireev, C. Rae Park, Y. Woo Park, and A. Yurgens, "Quantum hall effect in graphene decorated with disordered multilayer patches," Appl. Phys. Lett. 103, 233110 (2013).

${ }^{19}$ E. McCann, K. Kechedzhi, V. I. Fal'ko, H. Suzuura, T. Ando, and B. L. Altshuler, "Weak-localization magnetoresistance and valley symmetry in graphene," Phys. Rev. Lett. 97, 146805 (2006).

${ }^{20}$ J. Martin, N. Akerman, G. Ulbricht, T. Lohmann, J. H. Smet, K. von Klitzing, and A. Yacoby, "Observation of electron-hole puddles in graphene using a scanning single-electron transistor," Nat. Phys. 4, 144-148 (2008).

${ }^{21}$ J.-M. Poumirol, W. Escoffier, A. Kumar, B. Raquet, and M. Goiran, "Impact of disorder on the $\nu=2$ quantum hall plateau in graphene," Phys. Rev. B 82, 121401 (2010).

${ }^{22}$ A. A. Middleton and N. S. Wingreen, "Collective transport in arrays of small metallic dots," Phys. Rev. Lett. 71, 3198-3201 (1993). 
${ }^{23}$ D. Joung, L. Zhai, and S. I. Khondaker, "Coulomb blockade and hopping conduction in graphene quantum dots array," Phys. Rev. B 83, 115323 (2011).

${ }^{24}$ K. C. Beverly, J. F. Sampaio, and J. R. Heath, "Effects of size dispersion disorder on the charge transport in self-assembled 2-d Ag nanoparticle arrays," J. Phys. Chem. B 106, 2131-2135 (2002).

${ }^{25}$ E. C. Peters, A. J. M. Giesbers, and M. Burghard, "Variable range hopping in graphene antidot lattices," Phys. Status Solidi B 249, 2522-2525 (2012).

${ }^{26}$ A. J. M. Giesbers, E. C. Peters, M. Burghard, and K. Kern, "Charge transport gap in graphene antidot lattices," Phys. Rev. B 86, 045445 (2012).

${ }^{27}$ O. Entin-Wohlman, Y. Imry, and U. Sivan, "Orbital magnetoconductance in the variable-range-hopping regime," Phys. Rev. B 40, 8342-8348 (1989).

${ }^{28}$ V. L. Nguyen, B. Spivak, and B. Shklovskii, "Aaronov-bohm oscillations with normal and superconducting flux quanta in hopping conductivity," JETP Lett. 41, 42 (1985); available at http://www.jetpletters.ac.ru/ps/ 1440/article_21910.pdf.

${ }^{29}$ S.-T. Lo, C. Chuang, R. K. Puddy, T.-M. Chen, C. G. Smith, and C.-T. Liang, "Non-ohmic behavior of carrier transport in highly disordered graphene," Nanotechnology 24, 165201 (2013).

${ }^{30}$ N. V. Lien, "Crossovers in two-dimensional variable range hopping," Phys. Lett. A 207, 379-384 (1995).
${ }^{31}$ J. Guillemette, S. S. Sabri, B. Wu, K. Bennaceur, P. E. Gaskell, M. Savard, P. L. Lévesque, F. Mahvash, A. Guermoune, M. Siaj, R. Martel, T. Szkopek, and G. Gervais, "Quantum hall effect in hydrogenated graphene,” Phys. Rev. Lett. 110, 176801 (2013).

${ }^{32}$ C. Chuang, R. Puddy, H.-D. Lin, S.-T. Lo, T.-M. Chen, C. Smith, and C.T. Liang, "Experimental evidence for efrosshklovskii variable range hop ping in hydrogenated graphene," Solid State Commun. 152, 905-908 (2012).

${ }^{33}$ A. B. Kaiser, C. Gómez-Navarro, R. S. Sundaram, M. Burghard, and K. Kern, "Electrical conduction mechanism in chemically derived graphene monolayers," Nano Lett. 9, 1787-1792 (2009).

${ }^{34}$ H. Zhang, E. Bekyarova, J.-W. Huang, Z. Zhao, W. Bao, F. Wang, R. C. Haddon, and C. N. Lau, "Aryl functionalization as a route to band gap engineering in single layer graphene devices," Nano Lett. 11, 4047-4051 (2011).

${ }^{35}$ B. R. Matis, F. A. Bulat, A. L. Friedman, B. H. Houston, and J. W. Baldwin, "Giant negative magnetoresistance and a transition from strong to weak localization in hydrogenated graphene," Phys. Rev. B 85, 195437 (2012).

${ }^{36}$ M. F. Craciun, I. Khrapach, M. D. Barnes, and S. Russo, "Properties and applications of chemically functionalized graphene," J. Phys.: Condens. Matter 25, 423201 (2013). 